\title{
Simvastatin and sildenafil combine to attenuate pulmonary hypertension
}

\author{
L. Zhao*, A. Sebkhi*, O. Ali*, B. Wojciak-Stothard" ${ }^{\#}$ L. Mamanova*, Q. Yang*, \\ J. Wharton* and M.R. Wilkins*
}

ABSTRACT: Statins have been proposed to be a potential treatment for pulmonary arterial hypertension. If introduced into clinical practice, the statin would have to be used in conjunction with established therapy. We investigated the effects of combining simvastatin with a phosphodiesterase type-5 inhibitor, sildenafil, in the rat model of hypoxia-induced pulmonary hypertension.

Rats were allocated to either: 1) a prevention protocol, to receive simvastatin $20 \mathrm{mg} \cdot \mathrm{kg}^{-1} \cdot \mathrm{day}^{-1}$ by intraperitoneal injection or sildenafil $75 \mathrm{mg} \cdot \mathrm{kg}^{-1} \cdot \mathrm{day}^{-1}$ orally or the combination (or vehicle) for 2 weeks beginning at the start of exposure to hypoxia (10\% inspired oxygen); or 2 ) a treatment protocol, where the same agents were administered in the last 2 weeks of a 4-week period of hypoxia.

In both protocols, the combination of sildenafil and simvastatin lowered pulmonary artery pressure and produced a significantly greater reduction in right ventricular hypertrophy and pulmonary vascular muscularisation than either drug alone. Moreover, the combination augmented significantly endothelial nitric oxide synthase expression and cGMP levels in the lung and right ventricle above that produced by either drug independently and resulted in greater inhibition of RhoA activity.

These data suggest that simvastatin can be usefully combined with sildenafil in the treatment of pulmonary arterial hypertension to achieve greater therapeutic benefit.

KEYWORDS: Animal models, hypoxia, pulmonary hypertension, RhoA, sildenafil, simvastatin

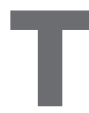
here is considerable interest in the pleiotropic effects of 3-hydroxy-3-methylglutaryl coenzyme A (HMG CoA) reductase inhibitors (statins) on the cardiovascular system beyond the benefits accrued from lowering cholesterol alone. This interest extends to pulmonary arterial hypertension (PAH) [1, 2]. While the treatment options for $\mathrm{PAH}$, particularly idiopathic PAH, have improved in recent years, with licensing approval granted to two orally active drugs, phosphodiesterase type-5 (PDE5) inhibitors and endothelin receptor antagonists, in addition to prostanoids, the outlook for PAH in its severest forms is still poor [3, 4]. New treatments are required.

In principle, statins may have a beneficial effect on the course of PAH on a number of levels. These include improving or restoring endothelial function, inhibiting the proliferation and migration of vascular smooth muscle cells, and decreasing vascular inflammation and oxidative stress [5-7]. Moreover, the effects of statins are not confined to the vasculature. Recent animal and human studies suggest that these drugs may also have direct beneficial effects on the myocardium [6]. Statins have been reported to reduce myocardial mass and fibrosis, increase capillary network density and attenuate electrical instability of the hypertrophied heart [8].

The pleiotropic effects of statins depend, in the main, on inhibiting the synthesis of the isoprenoid intermediates farnesylpyrophosphate and geranylgeranylpyrophosphate, which are essential for the post-translational isoprenylation, membrane localisation and activation of Ras and Rho small GTP-binding protein families respectively. These GTPases regulate many cellular functions and couple membrane growth factor receptors to intracellular pathways that affect cell proliferation [6, 7, 9]. RhoA and its downstream mediator Rho-kinase have become an attractive target for the treatment of PAH $[2,7]$. Rho-kinase inhibitors, such as fasudil, inhibit the development of pulmonary hypertension in experimental models [7]. Interestingly, cAMPand cGMP-dependent protein kinases have a key role in regulating Rho activation and expression in vascular smooth muscle cells $[10,11]$. Inhibition of
AFFILIATIONS

*Experimental Medicine and

Toxicology, Imperial College London,

Hammersmith Hospital, and

${ }^{\#}$ BHF Laboratories, Dept of

Medicine, University College London,

London, UK.

CORRESPONDENCE

L. Zhao

Experimental Medicine and

Toxicology

Imperial College London

Hammersmith Hospital

Ducane Road

London W12 ONN

UK

E-mail: I.zhao@imperial.ac.uk

Received:

Sept 192008

Accepted after revision:

March 262009 

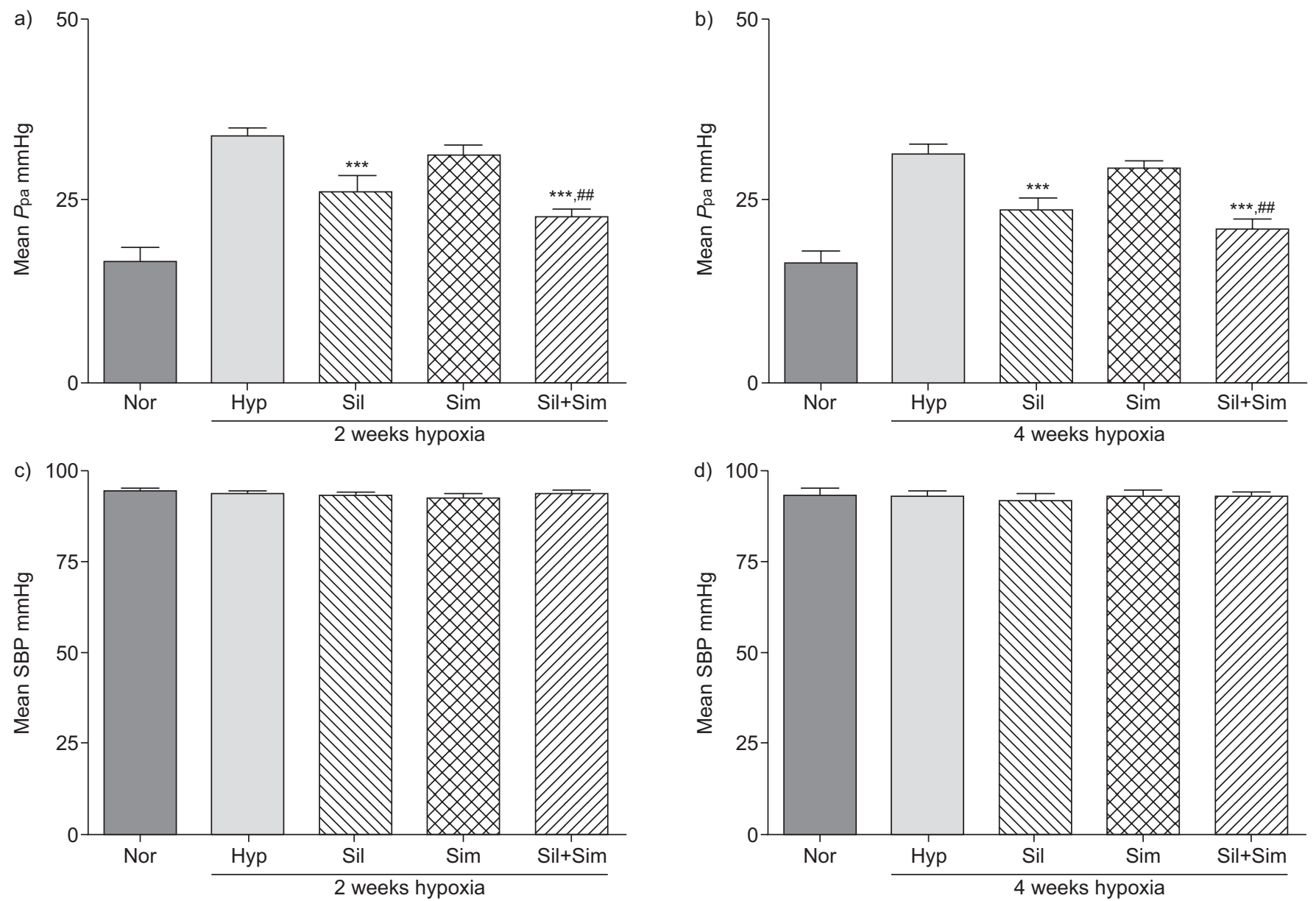

FIGURE 1. Mean pulmonary artery pressure (Ppa) and mean systolic blood pressure (SBP) in normoxic control rats (Nor) and animals exposed to 2 and 4 weeks of hypoxia (10\% oxygen) in the absence (Hyp) and presence of simvastatin $\left(25 \mathrm{mg} \cdot \mathrm{kg}^{-1} \cdot \mathrm{day}^{-1}\right)(\mathrm{Sim})$, sildenafil $\left(75 \mathrm{mg} \cdot \mathrm{kg}^{-1} \cdot \mathrm{day}^{-1}\right)$ (Sil) or sildenafil plus simvastatin combination (Sil+Sim). Exposure to chronic hypoxia for 2 and 4 weeks resulted in a doubling of $P$ pa $(33.5 \pm 1.6$ and $31.2 \pm 1.3$ compared to normoxia control $16.4 \pm 1.8 \mathrm{mmHg} ; \mathrm{p}<0.001)$. In both prevention ( $\mathrm{a}$ and $\mathrm{c}$ ) and treatment studies ( $\mathrm{b}$ and $\mathrm{d}$ ), sildenafil treatment reduced mean Ppa (prevention $26.1 \pm 2.3$ and treatment group $23.7 \pm 1.5 \mathrm{mmHg}$ ) significantly. Simvastatin alone had little effect (prevention $31.0 \pm 1.6$ and treatment group $29.5 \pm 1.0 \mathrm{mmHg}$ ), but was effective when combined with sildenafil (prevention $22.6 \pm 1.2$ and treatment group $20.9 \pm 1.7 \mathrm{mmHg}$ ), resulting in a mean $P$ pa comparable to normoxic controls (prevention $p=0.069$ and treatment group $p=0.149$, compared with normoxia control group). $n \geqslant 6$ for each group. ${ }^{* *}: p<0.001$ compared with hypoxia group. ${ }^{\# \#}: p<0.01$ compared with simvastatin group.

the RhoA/Rho-kinase signalling pathway may contribute, therefore, to the beneficial effect of established therapies, such as the PDE5 inhibitor sildenafil, in the treatment of pulmonary hypertension $[12,13]$.

Statins attenuate the development of pulmonary hypertension in experimental animal models [14-18] and have been reported to reverse established pulmonary hypertension and vascular remodelling, induced either by pneumonectomy and monocrotaline treatment [19] or chronic hypoxia [20]. This appears to be achieved through increased apoptosis as well as reduced proliferation of smooth muscle cells in obstructive vascular lesions $[17,19]$. An effect on the mobilisation and homing of bone marrow-derived progentor cells has also been proposed [21]. As a result, statins have become an attractive potential therapy for PAH. A recent uncontrolled observational study indicated that simvastatin is well tolerated in patients with different types of pulmonary hypertension, though it was not clear if improvement in their condition could be attributed to the drug treatment [22].
If statin therapy has a role in the treatment of $\mathrm{PAH}$, it will be used in combination with established treatment, not as a sole therapy. We examined, therefore, the effect of combining simvastatin with a common first choice therapy for PAH, sildenafil, on hypoxiainduced pulmonary hypertension in the rat. Specifically, we investigated the effects of this combination on pulmonary artery pressure $(\mathrm{Ppa})$, right ventricular hypertrophy $(\mathrm{RVH})$ and pulmonary vascular remodelling. In addition, we explored the likely mechanism of any interaction through the endothelial nitric oxide synthase (eNOS)-cGMP and RhoA signalling pathways.

\section{METHODS}

\section{Animals and experimental design}

Male Sprague-Dawley rats (280-400 g) from Charles River (Margate, UK) were used for all experiments. All studies were conducted in accordance with the UK Home Office Animals (Scientific Procedures) Act 1986 (London, UK).

Animals were divided into four groups (six to eight in each group) for each protocol: 1) normoxia control; 2) hypoxia 


\begin{tabular}{|c|c|c|c|c|c|c|c|c|}
\hline \multirow[t]{2}{*}{ Treatment } & \multicolumn{4}{|c|}{ Prevention study } & \multicolumn{4}{|c|}{ Treatment study } \\
\hline & Ppa & RVH & Remodelling & Hct & Ppa & RVH & Remodelling & Het \\
\hline Simvastatin & $15 \pm 6$ & $50 \pm 5$ & $29 \pm 5$ & $22 \pm 5$ & $12 \pm 6$ & $61 \pm 3$ & $26 \pm 5$ & $15 \pm 2$ \\
\hline
\end{tabular}

control (given intraperitoneal vehicle saline $0.2 \mathrm{~mL} \cdot \mathrm{rat}^{-1} \cdot \mathrm{day}^{-1}$ ); 3) hypoxia plus simvastatin $20 \mathrm{mg} \cdot \mathrm{kg}^{-1} \cdot \mathrm{day}^{-1}$ i.p. [14]; 4) hypoxia plus sildenafil $75 \mathrm{mg} \cdot \mathrm{kg}^{-1} \cdot \mathrm{day}^{-1}$ (in drinking water); and 5) hypoxia plus simvastatin and sildenafil in combination. Animals were weighed every other day and treatment doses were calculated by using the most recent average body weights for the group. Injection was carried out at approximately the same time each day. The sildenafil dose was monitored by weighing drinking bottles daily, and adjusting concentration to maintain dose if necessary. All animals had free access to food and water and were exposed to a normal $12 \mathrm{~h} / 12 \mathrm{~h}$ light/dark cycle.

Rats were exposed to chronic hypoxia in a $10 \%$ oxygen normobaric chamber as previously described [23]. Two distinct protocols were employed. In one, the prevention study protocol, animals were pre-treated for 2 days and treatment was continued during 2 weeks of exposure to hypoxia. In the other, the treatment study protocol, animals were exposed to hypoxia for 4 weeks, with treatment beginning after 2 weeks of hypoxia exposure.

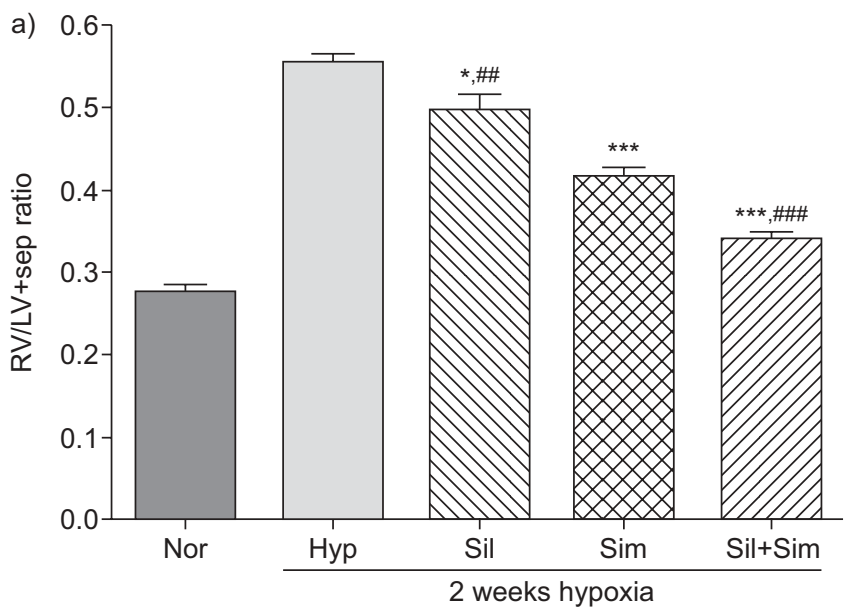

\section{Haemodynamic measurements}

At the end of the experiment, animals were weighed and anaesthetised (Hypnorm $0.25 \mathrm{~mL} \cdot \mathrm{kg}^{-1}$; midazolam $25 \mathrm{mg} \cdot \mathrm{kg}^{-1}$ i.p.). Ppa was measured via a pre-curved catheter inserted through the right jugular vein, passed by the right ventricle and into the pulmonary artery. Systemic blood pressure (SBP) was measured by cannulating the carotid artery. These fluid-filled catheters were connected to the pressure transducer of a PowerLab Data Acquisition system (ADInstruments Ltd, Chalgrove, UK) and pressure measurements were recorded from stable tracings.

Following sacrifice, plasma was collected, hearts were harvested and ventricular chambers dissected carefully and weighed. RVH was assessed from the ratio of the right ventricle to the left ventricle, plus the septum mass. The right lungs were snap frozen in liquid nitrogen and stored at $-80^{\circ} \mathrm{C}$ for biochemical measurements as detailed in the following sections. The left lungs were fixed by inflation with $10 \%$ formalin in phosphate-buffered saline, embedded in paraffin and sectioned for histology.

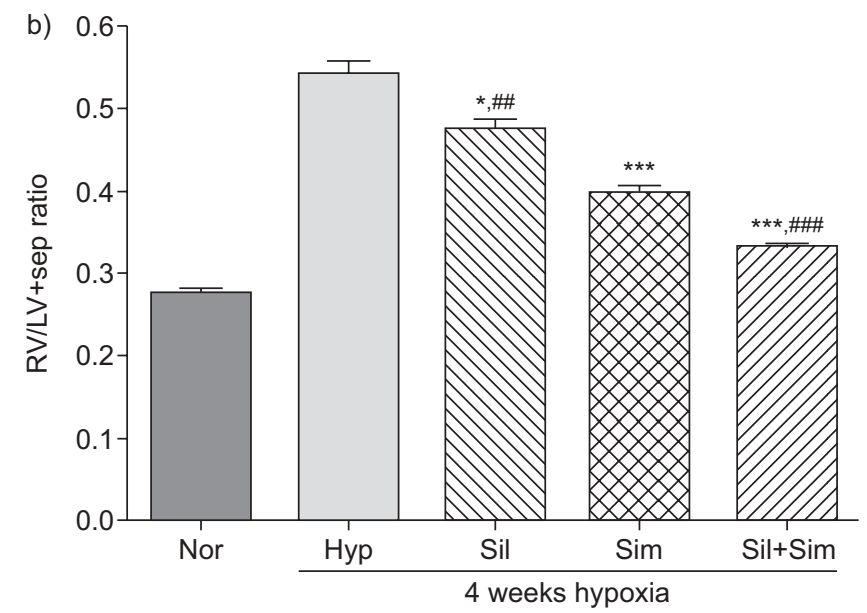

FIGURE 2. Right ventricular hypertrophy (RVH) in normoxic control rats (Nor) and animals exposed to 2 and 4 weeks of hypoxia (10\% oxygen) in the absence (Hyp) and presence of simvastatin $\left(25 \mathrm{mg} \cdot \mathrm{kg}^{-1} \cdot \mathrm{day}^{-1}\right)(\mathrm{Sim})$, sildenafil $\left(75 \mathrm{mg} \cdot \mathrm{kg}^{-1} \cdot \mathrm{day}^{-1}\right)$ (Sil) or sildenafil plus simvastatin combination (Sil+Sim). RVH was assessed as the ratio of the right ventricle to the left ventricle plus the septum mass (RV/LV+sep). Hypoxia induced significant RVH after both 2 and 4 weeks of hypoxia ( $0.56 \pm 0.02$ and $0.54 \pm 0.02$ compared with normoxia $0.28 \pm 0.01 ; p<0.001$ ). In both the a) prevention and b) treatment study groups, administration of sildenafil (prevention $0.49 \pm 0.02$ and treatment $0.48 \pm 0.01$ ) and simvastatin (prevention $0.41 \pm 0.01$ and treatment $0.40 \pm 0.01$ ) attenuated RVH significantly, the effect of simvastatin being significantly greater than that seen with sildenafil $(p<0.01)$. The effect was significantly enhanced following combined treatment with both drugs (prevention $0.33 \pm 0.01$ and treatment $0.33 \pm 0.01$ ). $n \geqslant 6$ for each group. *: $p<0.05$; ${ }^{* * *}: \mathrm{p}<0.001$ compared with hypoxia group. ${ }^{\# \#: ~} \mathrm{p}<0.01 ;{ }^{\# \#:} \mathrm{p}<0.001$ compared with simvastatin group. 

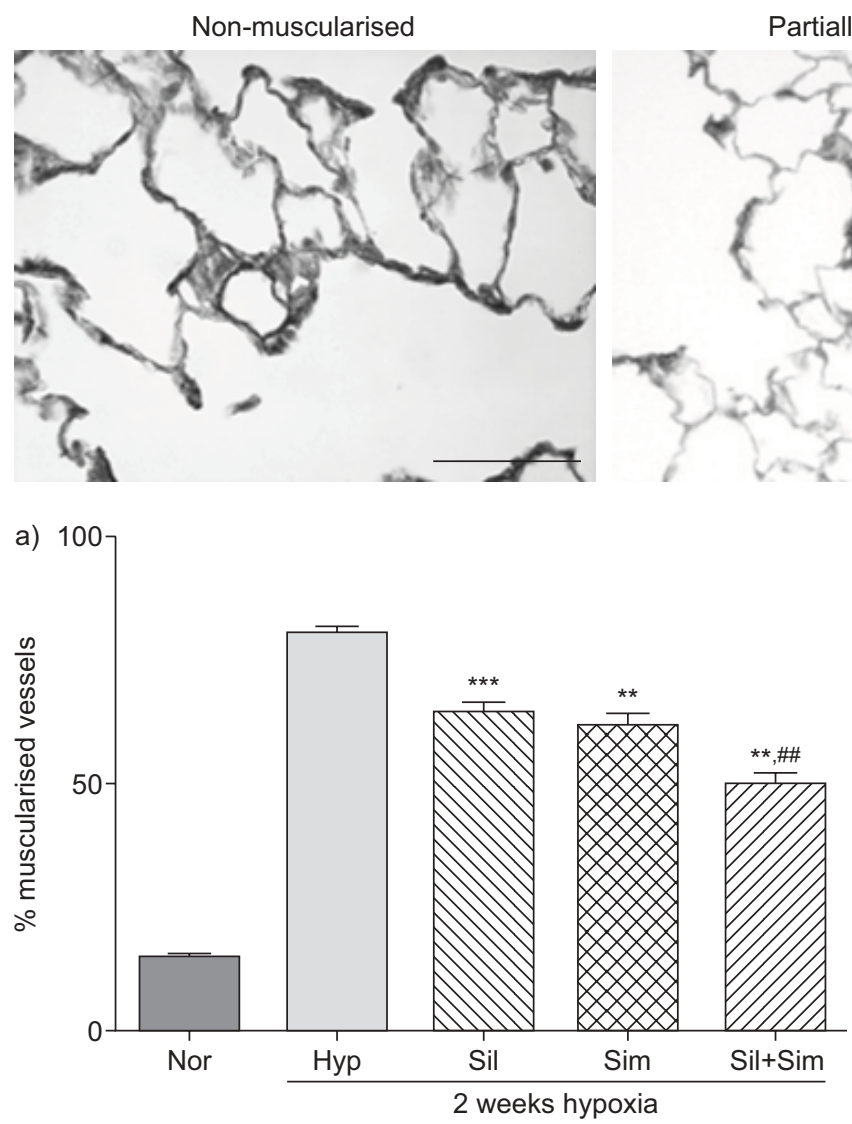
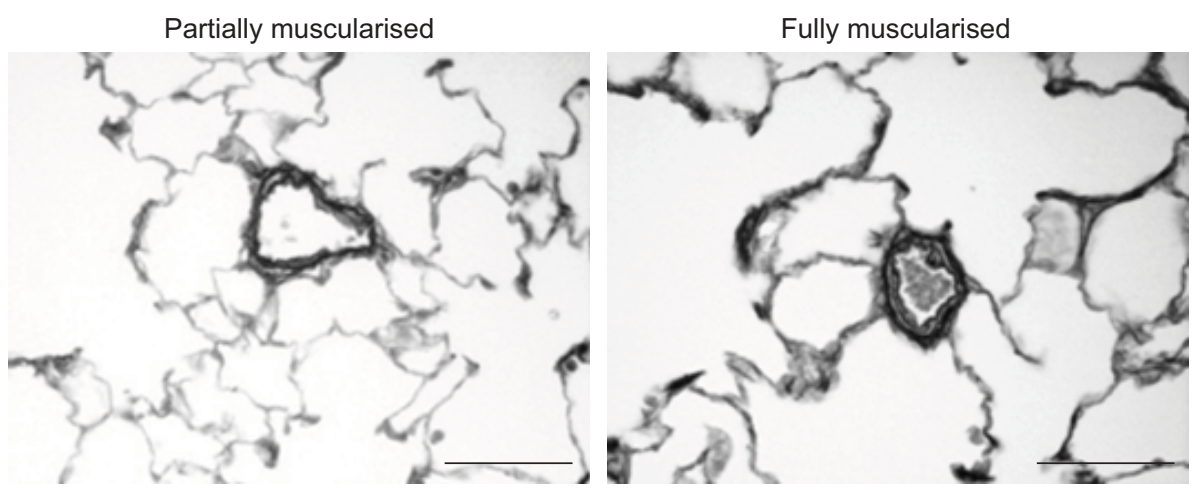

b) 100

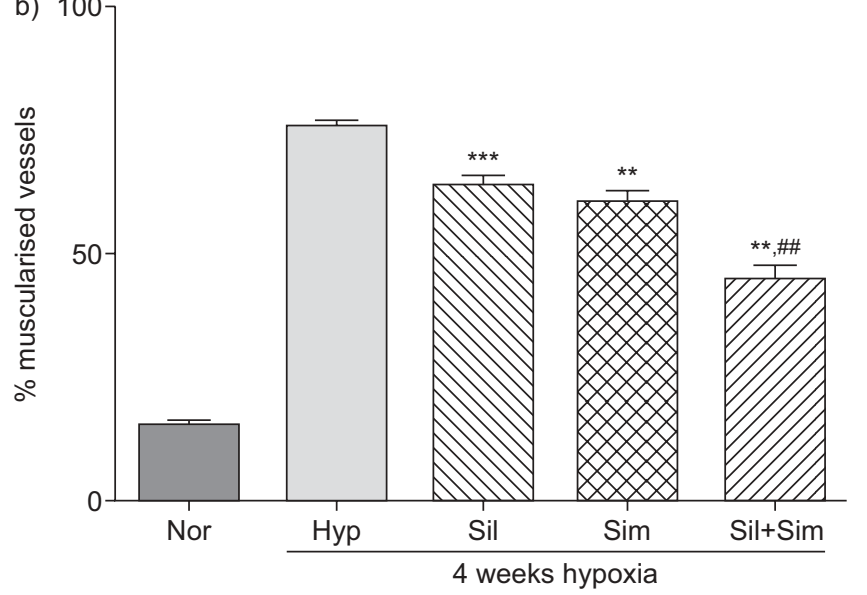

FIGURE 3. Percentage muscularised vessels in normoxic control rats (Nor) and animals exposed to 2 and 4 weeks hypoxia (10\% oxygen) in the absence (Hyp) and presence of simvastatin $\left(25 \mathrm{mg} \cdot \mathrm{kg}^{-1} \cdot\right.$ day $\left.^{-1}\right)\left(\right.$ Sim), sildenafil $\left(75 \mathrm{mg} \cdot \mathrm{kg}^{-1} \cdot\right.$ day $\left.^{-1}\right)$ (Sil) or sildenafil plus simvastatin combination (Sil+Sim). The proportion of muscularised pulmonary arteries increased markedly in rats exposed to hypoxia for 2 or 4 weeks $(75-80 \%)$ compared with normoxia controls (15 $\pm 0.7 \%)$. In the a) prevention and b) treatment study groups, sildenafil (prevention $61.9 \pm 3.3$ and treatment $63.3 \pm 2.0 \%$ ) and simvastatin (prevention $61.9 \pm 3.3$ and treatment $59.9 \pm 2.7 \%$ ) both prevented and reversed vascular remodelling and when administered together had a greater effect than either agent alone (prevention $49.5 \pm 2.9$ and treatment group $45.0 \pm 3.0 \%$ ). $* *: p<0.01 ;{ }^{* *}: p<0.001$ compared with hypoxia group. ${ }^{\# \#}: p<0.01$ compared with simvastatin group or sildenafil group. Scale bars $=50 \mu \mathrm{m}$.

\section{Histological analysis}

Histological assessment of vascular remodelling was performed as previously described [24]. Transverse lung sections were stained using van Gieson's elastic method. The proportion of vessels $<100 \mu \mathrm{m}$ in diameter, with double elastic laminae occupying $>50 \%$ of the circumference, was quantified in two separate sections from each animal and expressed as a percentage of total vessels counted (per cent muscularised vessels). Counting was performed blind to the treatment of the rat from which the sections were derived.

\section{eNOS Western blotting}

Lungs were homogenised in extract buffer $(50 \mathrm{mM}$ Tris- $\mathrm{HCl}$ pH 7.5, $150 \mathrm{mM} \mathrm{NaCl}, 0.1 \%$ SDS, $0.5 \%$ sodium deoxycholate, $1 \%$ non-idet P-40, $1 \mathrm{mM}$ PMSF, $1 \mathrm{mM}$ dithiothreitol, protease inhibitors). Equal amounts of protein lysates $(15 \mu \mathrm{g})$ were resolved using SDS-PAGE and transferred to nitrocellulose membranes (Immobilon (im-P; Millipore, Watford, UK). Membranes were incubated with a purified mouse monoclonal antibody against human eNOS amino acid 1025-1203 (Becton Dickinson UK Ltd, BD Diagnostics, Oxford, UK) diluted (1:1,000) in 6\% non-fat milk in PBS-Tween (PBS, $0.1 \%$ Tween 20) overnight at $4^{\circ} \mathrm{C}$. After washing $(6 \times 10 \mathrm{~min})$, they were incubated with horseradish peroxidase (HRP)-conjugated secondary antibodies, diluted $(1: 20,000)$ in $6 \%$ non-fat milk in PBS-Tween for $1 \mathrm{~h}$ at room temperature. They were then washed again and proteins visualised by chemiluminescence (SuperSignal; Pierce, Cramlington, UK). Optical densities of individual bands were measured. eNOS expression was normalised to $\beta$-actin for the lung and to $\alpha$-actin for the right ventricle. The data are presented as per cent mean of normoxic control values.

\section{Measurement of RhoA expression and activity}

An affinity-precipitation assay for endogenous GTP-loaded (active) Rho is commonly regarded as a reliable assay for the measurement of activated protein levels, which reflect tissue RhoA activity. GTP-loaded RhoA levels were measured with recombinant glutathione S-transferase-rhotekin Rho-binding domain (GST-RBD) bound to glutathione beads (GE Healthcare, Uppsala, Sweden), prepared as described previously [25]. Fragments of lung and right ventricle were homogenised, suspended in the ice-cold lysis buffer and spun down to remove cell debris. The detailed protocol of preparation 

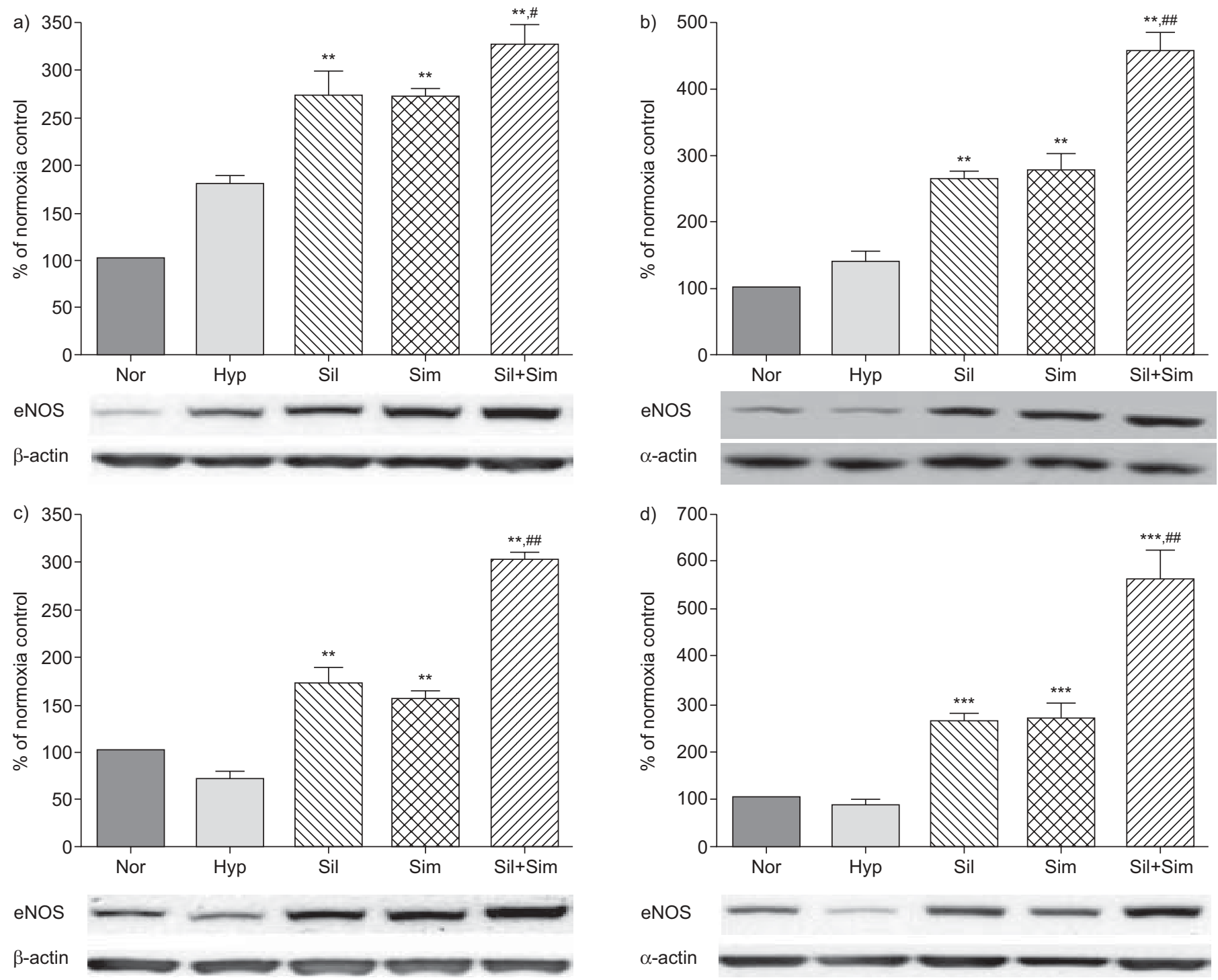

FIGURE 4. Endothelial nitric oxide synthase (eNOS) expression levels in the lung (a and c) and right ventricle (b and d). The data were generated from optical density measurements of individual bands from Western blots and normalised to $\beta$-actin for the lung and to $\alpha$-actin for the right ventricle. Changes in eNOS expression are presented as per cent mean of normoxia control values. a and b) Prevention study group; $c$ and d) treatment study group. Representative examples of Western blots of eNOS, $\beta$-actin and $\alpha$-actin are shown below the graphs. $\mathrm{n}=4$ for each group. Nor: normoxic controls; Hyp: hypoxia (10\% oxygen) group; Sil: sildenafil group; Sim: simvastatin group; Sil+Sim: sildenafil plus simvastatin combination. **: $p<0.01 ; * *: p<0.001$ compared with hypoxia group. ${ }^{*}: p<0.05 ;{ }^{* \#}: p<0.01$ compared with simvastatin or sildenafil group.

of lysis/wash buffer used in Rho pull-down assays was provided by Upstate Biotechnology, Watford, UK (Rho Activation Assay Kit). $100 \mu \mathrm{g}$ of GST-RBD was added to the supernatant and incubated with rotation for $60 \mathrm{~min}$ at $4^{\circ} \mathrm{C}$. The beads were collected by centrifugation, washed three times in the lysis/wash buffer, and the affinity-precipitated RhoA was resolved by SDS-PAGE and detected by Western blotting. A mouse monoclonal anti-RhoA antibody (Santa Cruz Biotechnology, Wembley, UK) was used at a dilution of 1:1,000 and a goat polyclonal anti-mouse immunoglobulin G HRPlabelled antibody (Dako, Ely, UK) at 1:2,000. GTP-RhoA expression levels were normalised to total RhoA protein expression levels and presented as per cent mean of normoxia control values.

\section{cGMP measurements}

Lung and right ventricular tissue samples were homogenised in cold $6 \%$ (weight/volume) trichloroacetic acid at $2-8^{\circ} \mathrm{C}$ containing IBMX $(2.5 \mathrm{mM})$. After centrifugation, supernatants were washed four times with water-saturated diethyl ether. The aqueous extracts were then lyophilised. Dried extracts were redissolved in the assay buffer and cGMP levels were measured by cGMP Enzymeimmunoassay Biotrak (EIA) System (GE Healthcare, Amersham, UK) according to the manufacturer's instructions.

\section{Statistics}

Data are expressed as the mean \pm SEM of individual values. Data were analysed using one-way ANOVA, followed by 

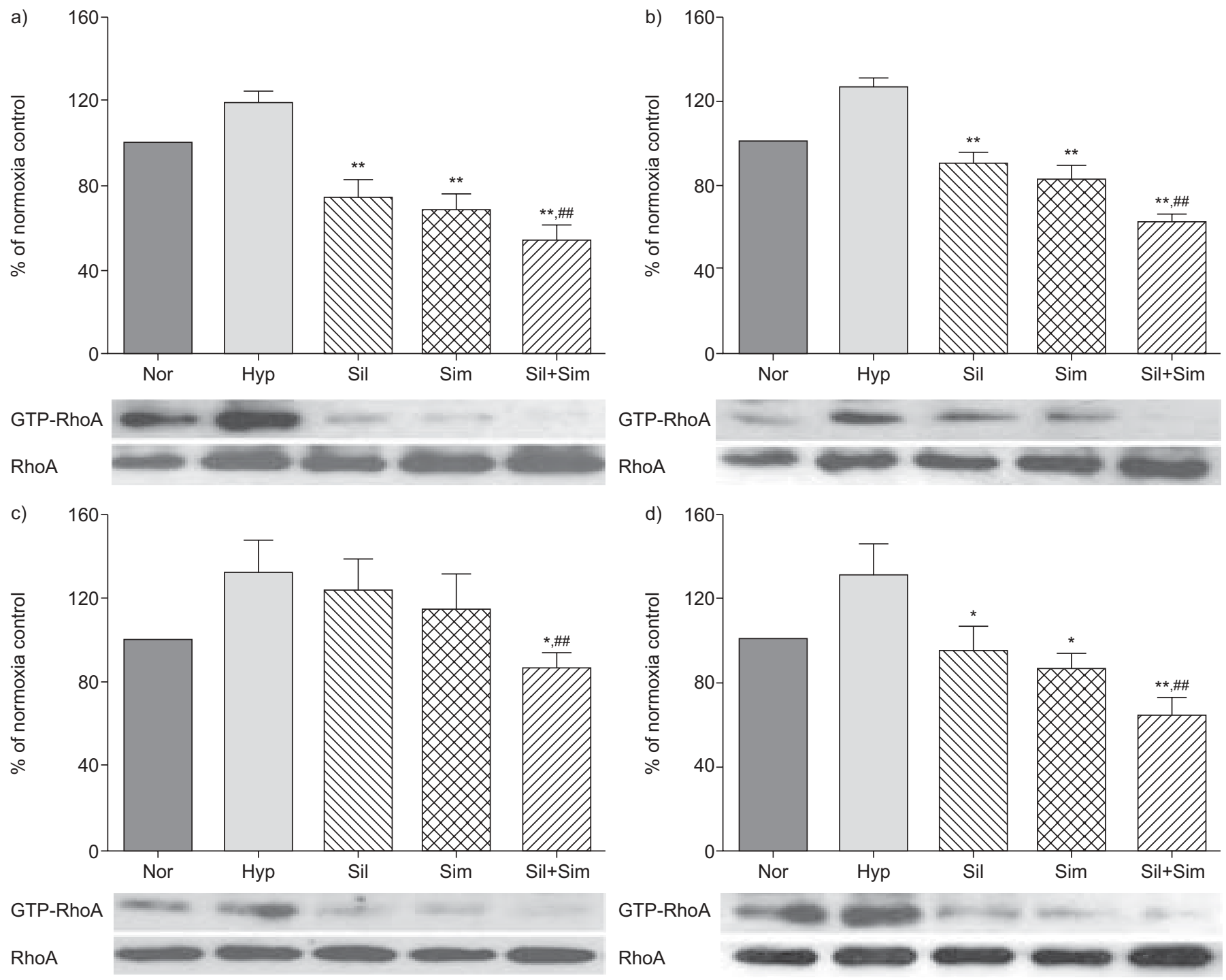

FIGURE 5. Effects on GTP-RhoA and RhoA expression levels in the lung (a and $c$ ) and right ventricle (b and d). GTP-RhoA levels (a measure of activated RhoA protein) was measured in affinity precipitation assays and resolved by Western blotting. The data were normalised to total RhoA protein and expressed as per cent mean of normoxia controls. a and b) Prevention study group; $\mathrm{c}$ and d) treatment study group. Representative examples of Western blots showing changes in GTP-RhoA and RhoA expression levels are shown below the graphs. $n=3$ for each group. Nor: normoxic controls; Hyp: hypoxia (10\% oxygen) group; Sil: sildenafil group; Sim: simvastatin group; Sil+Sim: sildenafil plus simvastatin combination. *: $p<0.05 ;{ }^{*}$ : $p<0.01$ compared with hypoxia group. ${ }^{\# \#}: p<0.01$ compared with simvastatin or sildenafil group.

Turkey's multiple comparison test or with unpaired t-test between two groups, to establish individual group differences. Statistical significance was set at $\mathrm{p}<0.05$.

\section{RESULTS}

\section{Prevention and treatment of hypoxia-induced pulmonary hypertension}

Exposure to chronic hypoxia for 2 weeks resulted in a two-fold increase in mean $P$ pa. This was attenuated in animals receiving sildenafil and the combination with simvastatin, but not with simvastatin alone (fig. 1a; table 1).

A similar increase in mean $P$ pa was observed after 4 weeks of exposure to hypoxia, consistent with our previous experience that the maximum effect is seen at around 2 weeks [23]. Treatment with sildenafil alone and in combination with simvastatin produced a marked reduction but simvastatin alone had little effect (fig. 1b; table 1). No significant effect was seen on SBP in either study protocol (fig. 1c and d).

\section{Prevention and treatment of hypoxia-induced right ventricular hypertrophy}

Hypoxia-vehicle treated animals showed significant RVH after both 2- and 4-week hypoxia (fig. 2). Administration of either sildenafil or simvastatin alone was accompanied by a significant reduction in RVH in both protocols and the effect was significantly enhanced following combined treatment with both drugs (fig. 2; table 1).

\section{Prevention and treatment of hypoxia-induced pulmonary vascular remodelling}

The proportion of muscularised pulmonary arterioles increased markedly in rats exposed to hypoxia for 2 or 4 weeks 

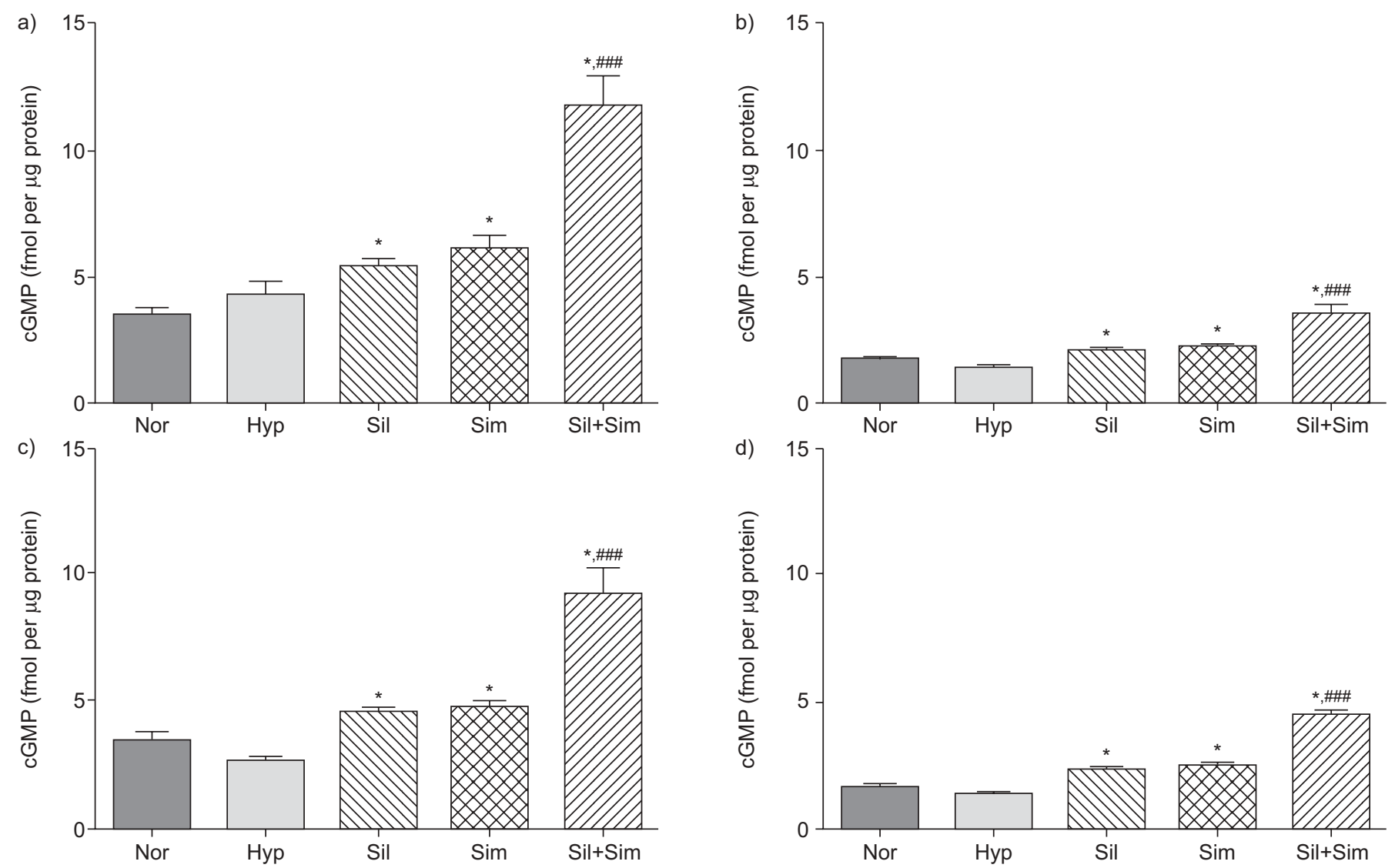

FIGURE 6. Effects on cGMP levels in the lung ( $a$ and $c$ ) and right ventricle ( $b$ and d) of normoxic control rats (Nor) and animals exposed to 2 and 4 weeks of hypoxia (10\% oxygen) in the absence (Hyp) and presence of simvastatin $\left(25 \mathrm{mg} \cdot \mathrm{kg}^{-1} \cdot \mathrm{day}^{-1}\right)(\mathrm{Sim})$, sildenafil $\left(75 \mathrm{mg} \cdot \mathrm{kg}^{-1} \cdot \mathrm{day}^{-1}\right)$ (Sil) or sildenafil plus simvastatin combination (Sil+Sim). a and b) Prevention study group; $c$ and d) treatment study group. *: $p<0.05$ compared with normoxia control and hypoxia group. ${ }^{\# \# \#}: p<0.001$ compared with all the other groups.

(to $75-80 \%)$ compared with normoxia controls $(15 \%$; $<<0.001)$ (fig. 3a-b). Sildenafil and simvastatin attenuated the development of muscularisation in both study protocols and administered together had a greater effect than either agent given alone (fig. 3a-b).

\section{Effects on haematocrit}

Hypoxia exposure for 2 and 4 weeks induced significant increases in haematocrit $(56 \pm 3 \%$ and $69 \pm 2 \% ; \mathrm{p}<0.001)$ compared with normoxia control $(40 \pm 1 \%)$. Treatment with simvastatin, but not sildenafil, was associated with a reduction in hypoxia-induced polycythaemia (table 1).

\section{Effects on eNOS expression levels}

Levels of eNOS protein expression in both the lung $(179 \pm 10 \%$; $\mathrm{p}<0.05)$ and right ventricle $(136 \pm 18 \% ; \mathrm{p}<0.05)$ were increased after 2 weeks of hypoxia exposure (fig. $4 a-b$ ). Sildenafil and simvastatin both induced a further rise in eNOS expression and when administered together had a greater effect than either agent alone (fig. 4a-b). Following 4 weeks of hypoxia exposure, eNOS levels were decreased in both the lung $(72 \pm 7 \% ; \mathrm{p}<0.05)$ and right ventricle $(85 \pm 10 \% ; \mathrm{p}<0.05)$ compared to normoxia controls. Treatment with sildenafil and simvastatin again induced a significant increase, which was enhanced further when the treatments were combined (fig. $4 \mathrm{c}-\mathrm{d}$ ).

\section{Effects on RhoA expression and activity}

Hypoxia exposure for 2 and 4 weeks induced an increase in GTP-RhoA expression levels in the lung and right ventricle compared with normoxic control tissues, without significant changes in RhoA protein expression (fig. 5). In the lung tissues from the prevention protocol, sildenafil or simvastatin alone significantly reduced GTP-RhoA expression levels compared with hypoxic controls, while the combination treatment had the strongest effect (fig. 5a). Similarly in the right ventricle, single treatment with sildenafil or simvastatin was sufficient to reduce GTP-RhoA expression levels to normoxia control levels, while the combination treatment $(80 \pm 4 \%)$ significantly reduced GTP-RhoA expression levels below that measured in animals in a normal atmosphere (fig. 5b). In the treatment protocol, single treatment with sildenafil or simvastatin reduced GTP-RhoA expression levels in the right ventricle, and the combination treatment had the greatest effect (fig. $5 c-d$ ).

\section{Effects on cGMP levels}

The levels of cGMP were not significantly affected by chronic hypoxia following 4 weeks of exposure (fig. 6). Sildenafil and 
simvastatin both induced a modest increase in cGMP levels in the lung and right ventricle and a marked two- to three-fold rise was observed when the two drugs were administered together (fig. 6).

\section{DISCUSSION}

The current approach to the treatment of PAH is to combine drugs from different classes in the expectation of achieving greater therapeutic benefit. This study provides compelling evidence to suggest that the combination of sildenafil and simvastatin has therapeutic potential. In a rat model used in the pre-clinical assessment of all drug therapies approved for $\mathrm{PAH}$ to date, the combination of simvastatin and sildenafil, whether given at the start of exposure to hypoxia or after 2 weeks of hypoxia-induced pulmonary hypertension, was associated with a greater reduction in $\mathrm{RVH}$ and pulmonary vascular remodelling than seen with either drug alone. The combination also showed specificity for the pulmonary circulation. Moreover, these responses were accompanied by a more pronounced increase in lung and right ventricular eNOS expression and cGMP levels and greater reduction in GTP-RhoA levels (a marker of activated RhoA protein) in these tissues: biochemical effects consistent with the known actions of PDE5 and HMG CoA reductase inhibition.

Simvastatin has already been investigated in a number of different experimental models of pulmonary hypertension. GIRGIS and co-workers [14, 20] have shown previously that simvastatin $\left(20 \mathrm{mg} \cdot \mathrm{kg}^{-1} \cdot \mathrm{day}^{-1}\right)$ both prevented and regressed hypoxia-induced pulmonary hypertension, $\mathrm{RVH}$ and vascular remodelling in rats. NISHIMURA and co-workers [17, 19] reported that simvastatin $2 \mathrm{mg} \cdot \mathrm{kg}^{-1} \cdot \mathrm{day}^{-1}$ orally both prevented and reversed pulmonary hypertension and neointima formation and improved survival in a challenging rat model in which pulmonary hypertension is induced by monocrotaline and pneumonectomy. Recently, MCMurTRY et al. [26] found simvastatin $\left(2 \mathrm{mg} \cdot \mathrm{kg}^{-1} \cdot \mathrm{day}^{-1}\right.$ by gavage) less impressive in the monocrotaline model, retarding the progression of pulmonary hypertension and RVH at 4 weeks but with no effect on survival. TARASEVICIENE-STEWART et al. [27] have reported that simvastatin $\left(10 \mathrm{mg} \cdot \mathrm{kg}^{-1} \cdot\right.$ day $^{-1}$ by gavage) led to a significant improvement of the aggressively proliferative form of severe pulmonary hypertension induced by chronic vascular endothelial growth factor receptor blockade (SU5416) in combination with chronic hypoxia. The dose of simvastatin used in our study was at the higher end of the range used in previous studies. Despite this, used alone, we observed modest reductions in $P$ pa and hypoxia-induced polycythaemia compared with these reports $[14,20]$. There was a marked effect on $\mathrm{RVH}$ and vascular remodelling, although it was not fully reversed. It is possible that the lack of effect on $P$ pa is masked by reduced pulmonary vascular resistance and an improvement in cardiac output, but neither was measured in this study.

The more pronounced effect of simvastatin on RVH and vascular structure compared with $P$ pa may reflect a direct antitrophic action and its lack of vasoactive properties, although the relative contribution of vasoconstriction and vascular remodelling to hypoxia-induced pulmonary hypertension is the subject of debate [28]. Sildenafil, however, is a more effective vasorelaxant agent, with some antiproliferative properties. Sildenafil has been previously shown to be effective in the rat hypoxia model of pulmonary hypertension but does not regress $P$ pa, $\mathrm{RVH}$ and pulmonary vascular remodelling to normal [23]. The addition of simvastatin to sildenafil treatment maintained the reduction in $P$ pa and demonstrated additional antitrophic effects. Significantly, the dose of sildenafil used in this study $\left(75 \mathrm{mg} \cdot \mathrm{kg}^{-1} \cdot \mathrm{day}^{-1}\right.$ orally) is at or near the top of the dose-response curve for this drug in the rat hypoxia model, producing no greater reduction in $P$ pa than $25 \mathrm{mg} \cdot \mathrm{kg}^{-1} \cdot \mathrm{day}^{-1}$. Since increasing the dose of sildenafil has no further effect, the additional benefit gained from combination with simvastatin most likely reflects a true pharmacodynamic effect.

The cardiovascular effects of sildenafil are mediated mainly by cGMP. Both sildenafil and simvastatin increased eNOS expression and the combination of the two drugs had a more marked effect on eNOS expression and a greater than additive effect on tissue cGMP levels. In addition, combination of the two drugs markedly reduced GTP-RhoA expression. Levels of activated RhoA protein were significantly increased in both the lung and right ventricle after 2 and 4 weeks of hypoxia, in agreement with published data $[12,29]$. Both simvastatin and sildenafil attenuated GTP-RhoA expression in the lung and right ventricle during exposure to hypoxia, an effect augmented by combining the two drugs. RhoA is considered to be the main upstream activator of Rho kinase. RhoA may contribute to pulmonary hypertension by mediating the effects of vasoconstrictors such as angiotensin-II, endothelin-1 and acetylcholine and/or downregulating eNOS expression and nitric oxide production in endothelial cells [30,31]. RhoA and Rho kinase are important for the differentiation and survival of vascular smooth muscle cells and prolonged inhibition induces apoptosis in these cells in vitro [32]. Rho proteins can also regulate extracellular matrix production in various cell types [33]. RhoA has become a target for drug development in pulmonary hypertension [34]. Intravenous administration of the Rho kinase inhibitors fasudil or Y-27632 attenuated pulmonary hypertension and pulmonary vascular remodelling and enhanced eNOS expression in monocrotaline and chronic hypoxia-induced $\mathrm{PH}$ in rodents [35-37]. Interestingly fasudil was less effective in reducing chronic hypoxia-induced pulmonary hypertension in eNOS-deficient mice compared with wild-type animals, suggesting Rho kinase signalling is eNOS dependent [37]. Furthermore, the cardioprotective effects of statins also appear to involve eNOS [38, 39]. Our data would support the thesis that eNOS-RhoA-cGMP is a convergent signalling pathway mediating the combined, augmented effects of sildenafil and simvastatin on $P$ pa, RVH and vascular remodelling in this rat model of pulmonary hypertension.

There are a number of limitations to this study. First, it is not clear whether we have achieved the maximum pharmacological effects of simvastatin in our hypoxia model. Specifically, the effects of simvastatin on $P$ pa and polycythaemia were less marked than those previously reported, despite using the same dose of simvastatin [14, 20]. However, we did note some external surface granularity of the liver in the simvastatin treated animals, suggesting we were close to a dose that might cause liver toxicity. Furthermore, we did not assess pulmonary vascular resistance and cardiac output. Thirdly, a pharmacokinetic 
interaction between simvastatin and sildenafil needs to be investigated in future studies.

In summary, the addition of simvastatin to sildenafil has a significant protective effect and significantly reverses hypoxiainduced pulmonary hypertension and remodelling. The effects, particularly the trophic effects on RVH and vascular remodelling, are more pronounced when the drugs are combined than when given alone. This response may be explained, at least in part, by an additive effect on eNOS expression and cGMP levels in the lung and right ventricle, and a more marked inhibition of RhoA activity.

\section{SUPPORT STATEMENT}

This work was supported by the British Heart Foundation.

\section{STATEMENT OF INTEREST}

Statements of interest for J. Wharton and M.R. Wilkins can be found at www.erj.ersjournals.com/misc/statements.dtl

\section{REFERENCES}

1 Archer SL, Michelakis ED. An evidence-based approach to the management of pulmonary arterial hypertension. Curr Opin Cardiol 2006; 21: 385-392.

2 Ali O, Wharton J, Gibbs JS, et al. Emerging therapies for pulmonary arterial hypertension. Expert Opin Invest Drugs 2007; 16: 803-818.

3 Chin KM, Rubin LJ. Pulmonary arterial hypertension. J Am Coll Cardiol 2008; 22, 51: 1527-1538.

4 National Pulmonary Hypertension Centres of the UK and Ireland. Consensus statement on the management of pulmonary hypertension in clinical practice in the UK and Ireland. Thorax 2008; 63: Suppl. 2, ii1-ii41.

5 Wolfrum S, Jensen KS, Liao JK. Endothelium-dependent effects of statins. Arterioscler Thromb Vasc Biol 2003; 23: 729-736.

6 Liao JK, Laufs U. Pleiotropic effects of statins. Ann Rev Pharmacol Toxicol 2005; 45: 89-118.

7 Loirand G, Guerin P, Pacaud P. Rho kinases in cardiovascular physiology and pathophysiology. Circ Res 2006; 98: 322-334.

8 Simko F. Statins: a perspective for left ventricular hypertrophy treatment. Eur J Clin Invest 2007; 37: 681-691.

9 Carlin CM, Peacock AJ, Welsh DJ. Fluvastatin inhibits hypoxic proliferationand p38 MAPK activity in pulmonary artery fibroblasts. Am J Respir Cell Mol Biol 2007; 37: 447-456.

10 Sauzeau V, Le Jeune H, Cario-Toumaniantz C, et al. Cyclic GMPdependent protein kinase signaling pathway inhibits RhoAinduced $\mathrm{Ca}^{2+}$ sensitization of contraction in vascular smooth muscle. J Biol Chem 2000; 275: 21722-21729.

11 Sauzeau V, Rolli-Derkinderen M, Marionneau C, et al. RhoA expression is controlled by nitric oxide through cGMP-dependent protein kinase activation. J Biol Chem 2003; 278: 9472-9480.

12 Guilluy C, Sauzeau V, Rolli-Derkinderen M, et al. Inhibition of RhoA/Rho kinase pathway is involved in the beneficial effect of sildenafil on pulmonary hypertension. Br J Pharmacol 2005; 146: 1010-1018.

13 Sauzeau V, Rolli-Derkinderen M, Lehoux S, et al. Sildenafil prevents change in RhoA expression induced by chronic hypoxia in rat pulmonary artery. Circ Res 2003; 93: 630-637.

14 Girgis RE, Li D, Zhan X, et al. Attenuation of chronic hypoxic pulmonary hypertension by simvastatin. Am J Physiol Heart Circ Physiol 2003; 285: H938-H945.

15 Guerard P, Rakotoniaina Z, Goirand F, et al. The HMG-CoA reductase inhibitor, pravastatin, prevents the development of monocrotaline-induced pulmonary hypertension in the rat through reduction of endothelial cell apoptosis and overexpression of eNOS. Naunyn Schmiedebergs Arch Pharmacol 2006; 373: 401-414.

16 Murata T, Kinoshita K, Hori M, et al. Statin protects endothelial nitric oxide synthase activity in hypoxia-induced pulmonary hypertension. Arterioscler Thromb Vasc Biol 2005; 25: 2335-2342.

17 Nishimura T, Faul JL, Berry GJ, et al. Simvastatin attenuates smooth muscle neointimal proliferation and pulmonary hypertension in rats. Am J Respir Crit Care Med 2002; 166: 1403-1408.

18 Taraseviciene-Stewart L, Scerbavicius R, Choe KH, et al. Simvastatin causes endothelial cell apoptosis and attenuates severe pulmonary hypertension. Am J Physiol Lung Cell Mol Physiol 2006; 291: L668-L676.

19 Nishimura T, Vaszar LT, Faul JL, et al. Simvastatin rescues rats from fatal pulmonary hypertension by inducing apoptosis of neointimal smooth muscle cells. Circulation 2003; 108: 1640-1645.

20 Girgis RE, Mozammel S, Champion HC, et al. Regression of chronic hypoxic pulmonary hypertension by simvastatin. Am J Physiol Lung Cell Mol Physiol 2007; 292: L1105-L1110.

21 Satoh K, Fukumoto Y, Nakano M, et al. Statin ameliorates hypoxiainduced pulmonary hypertension associated with down-regulated stromal cell-derived factor-1. Cardiovasc Res 2009; 81: 226-234.

22 Kao PN. Simvastatin treatment of pulmonary hypertension: an observational case series. Chest 2005; 127: 1446-1452.

23 Sebkhi A, Strange JW, Phillips SC, et al. Phosphodiesterase type 5 as a target for the treatment of hypoxia-induced pulmonary hypertension. Circulation 2003; 107: 3230-3235.

24 Zhao L, Mason NA, Strange JW, et al. Beneficial effects of phosphodiesterase 5 inhibition in pulmonary hypertension are influenced by natriuretic peptide activity. Circulation 2003; 107: 234-237.

25 Ren XD, Schwartz MA. Regulation of inositol lipid kinases by Rho and Rac. Curr Opin Genet Dev 1998; 8: 63-67.

26 McMurtry MS, Bonnet S, Michelakis ED, et al. Statin therapy, alone or with rapamycin, does not reverse monocrotaline pulmonary arterial hypertension: the rapamcyin-atorvastatin-simvastatin study. Am J Physiol Lung Cell Mol Physiol 2007; 293: L933-L940.

27 Taraseviciene-Stewart L, Scerbavicius $\mathrm{R}$, Choe $\mathrm{KH}$, et al. Simvastatin causes endothelial cell apoptosis and attenuates severe pulmonary hypertension. Am J Physiol Lung Cell Mol Physiol 2006; 291: L668-L676.

28 Stenmark KR, McMurtry IF. Vascular remodelling versus vasoconstriction in chronic hypoxic pulmonary hypertension: a time for reappraisal? Circ Res 2005; 97: 95-98.

29 Jernigan NL, Walker BR, Resta TC. Chronic hypoxia augments protein kinase $\mathrm{G}$-mediated $\mathrm{Ca}^{2+}$ desensitization in pulmonary vascular smooth muscle through inhibition of RhoA/Rho kinase signaling. Am J Physiol Lung Cell Mol Physiol 2004; 287: L1220L1229.

30 Wojciak-Stothard B. New drug targets for pulmonary hypertension: Rho GTPases in pulmonary vascular remodelling. Postgrad Med J 2008; 84: 348-353.

31 Oka M, Fagan KA, Jones PL, et al. Therapeutic potential of RhoA/ Rho kinase inhibitors in pulmonary hypertension. $\mathrm{Br}$ J Pharmacol 2008; 155: 444-454.

32 Mack CP, Somlyo AV, Hautmann M, et al. Smooth muscle differentiation marker gene expression is regulated by RhoAmediated actin polymerization. J Biol Chem 2001; 276: 341-347.

33 Yoneda A, Ushakov D, Multhaupt HA, et al. Fibronectin matrix assembly requires distinct contributions from Rho kinases I and -II. Mol Biol Cell 2007; 18: 66-75.

34 Fukumoto Y, Tawara S, Shimokawa H. Recent progress in the treatment of pulmonary arterial hypertension: expectation for Rho-kinase inhibitors. Tohoku J Exp Med 2007; 211: 309-320.

35 Abe K, Shimokawa H, Morikawa K, et al. Long-term treatment with a Rho-kinase inhibitor improves monocrotaline-induced fatal pulmonary hypertension in rats. Circ Res 2004; 20, 94: 385-393. 
36 Fagan KA, Oka M, Bauer NR, et al. Attenuation of acute hypoxic pulmonary vasoconstriction and hypoxic pulmonary hypertension in mice by inhibition of Rho-kinase. Am J Physiol Lung Cell Mol Physiol 2004; 287: L656-L664.

37 Abe K, Tawara S, Oi K, et al. Long-term inhibition of Rho-kinase ameliorates hypoxia-induced pulmonary hypertension in mice. J Cardiovasc Pharmacol 2006; 48: 280-285.
38 Laufs U, Liao JK. Targeting Rho in cardiovascular disease. Circ Res 2000; 87: 526-528.

39 Landmesser U, Engberding N, Bahlmann FH, et al. Statin-induced improvement of endothelial progenitor cell mobilization, myocardial neovascularization, left ventricular function, and survival after experimental myocardial infarction requires endothelial nitric oxide synthase. Circulation 2004; 110: 1933-1939. 